\title{
STABILITY IN THE ISOPERIMETRIC PROBLEM FOR CONVEX OR NEARLY SPHERICAL DOMAINS IN $\mathbf{R}^{n}$
}

\author{
BENT FUGLEDE \\ Dedicated to the memory of Werner Fenchel
}

\begin{abstract}
For convex bodies $D$ in $\mathbf{R}^{n}$ the deviation $d$ from spherical shape is estimated from above in terms of the (dimensionless) isoperimetric deficiency $\Delta$ of $D$ as follows: $d \leq f(\Delta)$ (for $\Delta$ sufficiently small). Here $f$ is an explicit elementary function vanishing continuously at 0 . The estimate is sharp as regards the order of magnitude of $f$. The dimensions $n=2$ and 3 present anomalies as to the form of $f$. In the planar case $n=2$ the result is contained in an inequality due to T. Bonnesen. A qualitative consequence of the present result is that there is stability in the classical isoperimetric problem for convex bodies $D$ in $\mathbf{R}^{n}$ in the sense that, as $D$ varies, $d \rightarrow 0$ for $\Delta \rightarrow 0$. The proof of the estimate $d \leq f(\Delta)$ is based on a related estimate in the case of domains (not necessarily convex) that are supposed a priori to be nearly spherical in a certain sense.
\end{abstract}

\section{INTRODUCTION}

For a planar domain $D$ of area $A$, bounded by a simple closed curve $\partial D$ of length $L$, Bonnesen [2] showed in 1924 that $D$ has concentric inscribed and circumscribed circles of radii $r_{1}, r_{2}$ satisfying the inequality

$$
\left(r_{2}-r_{1}\right)^{2} \leq \frac{L^{2}}{4 \pi}-A .
$$

He also showed that the equality sign only occurs for a circle, and furthermore that the inequality ceases to hold in general if the right-hand member is multiplied by a constant $c<1$. Earlier, Bernstein [1] had obtained an equivalent inequality, though with a certain constant factor $c(\approx 1700)$ on the right, as a limit case of a corresponding inequality for domains on a sphere in $\mathbf{R}^{3}$.

Received by the editors May 13, 1988 and, in revised form, January 24, 1989. Presented August 23, 1988 to the 20th Nordic Congress of Mathematicians, Trondheim, Norway.

1980 Mathematics Subject Classification (1985 Revision). Primary 52A40, 52A20, 52A15; Secondary $49 \mathrm{~F} 22$.

Key words and phrases. Isoperimetric deficiency, convex bodies, nearly spherical domains, stability.

' Still another proof-quite short-can be read off from Hurwitz' Fourier series proof of the isoperimetric inequality in the plane [8], and that leads in a certain sense to a stronger inequality, involving a Sobolev 1-norm of the deviation of $\partial D$ from a circle, again estimated in terms of $L^{2}-4 \pi A$, see [6]. It also leads to Bonnesen's inequality with a constant factor $c(=5 \pi)$ on the right. 
The Bernstein-Bonnesen inequality implies of course the isoperimetric inequality $L^{2}-4 \pi A \geq 0$ with equality only for a circle, but it shows moreover that there is stability in the isoperimetric problem, in the sense that any domain $D$ with a small isoperimetric deficiency deviates only slightly from a circular disc.

The question naturally arises to which extent there is stability in the isoperimetric problem in higher dimensions. While in dimension 2 the question of stability reduces immediately to the case of convex domains (by passing to the convex hull, whereby the area increases while the length decreases), the situation in higher dimensions is quite different for convex domains and for general domains.

Let $\omega_{n}$ denote the volume, and hence $n \omega_{n}$ the surface area, of the unit ball $\Omega$ in $\mathbf{R}^{n}$. For a compact domain $D$ in $\mathbf{R}^{n}$ with Lipschitz class boundary $\partial D$ we denote by

$$
V=\omega_{n} v^{n}, \quad S=n \omega_{n} s^{n-1}
$$

the volume of $D$ and the area of $\partial D$, respectively. ${ }^{2}$ The isoperimetric deficiency $\Delta$ of $D$ will be defined as the dimensionless quantity

$$
\Delta=\frac{S}{n \omega_{n}}\left(\frac{V}{\omega_{n}}\right)^{-\frac{n-1}{n}}-1=\left(\frac{s}{v}\right)^{n-1}-1 .
$$

The isoperimetric inequality asserts that $\Delta \geq 0$, with equality only for a ball. ${ }^{3}$

It will be understood moreover that $D$ is "starshaped" with respect to its barycentre, which we may take to be 0 . After a change of scale we may assume that $V=\omega_{n}$. With these two normalizations the boundary $\partial D$ can be represented in polar coordinates $R \in \mathbf{R}_{+}, \xi \in \Sigma$ (the unit sphere in $\mathbf{R}^{n}$ ) by an equation

$$
R=1+u(\xi), \quad \xi \in \Sigma .
$$

In $\S 1$ we further suppose that $D$ is "nearly spherical" in the sense that

$$
\|u\|_{\infty} \leq \frac{3}{20 n}, \quad\|\nabla u\|_{\infty} \leq \frac{1}{2},
$$

where $\|\cdot\|_{p}, 1 \leq p \leq \infty$, denotes the $L^{p}$-norm with respect to the normalized surface measure on $\Sigma$. With this ad hoc restriction we show that there is stability in the isoperimetric problem in $\mathbf{R}^{n}$, both in Sobolev 1-norm and in uniform norm. More precisely, we obtain the following estimates, valid under the hypothesis $(*)$ :

$$
\frac{1}{10}\left(\|u\|_{2}^{2}+\|\nabla u\|_{2}^{2}\right) \leq \Delta \leq \frac{3}{5}\|\nabla u\|_{2}^{2}
$$

\footnotetext{
2 We shall include the case $n=2$, mainly because the planar case of (I.a) above is not covered by Bonnesen's work.

${ }^{3}$ The particular choice $n-1$ of a (positive) exponent of $s / v$ in the definition of $\Delta$ is of course immaterial, but it is convenient for the proof of (I.a) above.
} 


$$
\|u\|_{\infty}^{n-1} \leq \begin{cases}A \Delta^{\frac{1}{2}} & \text { for } n=2, \\ A \Delta \log \frac{A^{\prime}\|\nabla u\|_{\infty}^{2}}{\Delta} & \text { for } n=3, \Delta>0, \\ A \Delta\|\nabla u\|_{\infty}^{n-3} & \text { for } n \geq 4,\end{cases}
$$

where $A$ and $A^{\prime}$ are constants depending only on $n$ and explicity calculable (Theorem 1.2). In the cases $n \geq 3$ one may of course insert $\|\nabla u\|_{\infty} \leq 1 / 2$, cf. (*) above.

The stated equivalence between the square root of the isoperimetric deficiency $\Delta$ of a domain $D$ satisfying $(*)$ and the Sobolev 1-norm of $u$, as expressed in (I.a), was established in [6] for the case $n=3$ by almost the same method as in the present paper, and it was shown that some restriction like (*) above is necessary for stability-whether in uniform norm or in Sobolev 1 -norm as in (I.a). (An example is obtained e.g. by adding a thin "spike" to a ball.)

In $\S 2$ we specialize to the class of convex bodies $D$ in $\mathbf{R}^{n}$, and we show that within this class there is stability without any additional assumption like (*) above. More precisely it is shown (Theorem 2.3) that there exist explicitly calculable positive constants $\eta$ and $C$, depending only on $n$, such that any convex body $D$ in $\mathbf{R}^{n}$ with isoperimetric deficiency $\Delta<\eta$ is nearly spherical in the sense of $(*)$ and satisfies

$$
\|u\|_{\infty} \leq \begin{cases}C \Delta^{\frac{1}{2}} & \text { for } n=2, \\ C\left(\Delta \log \frac{1}{\Delta}\right)^{\frac{1}{2}} & \text { for } n=3, \\ C \Delta^{\frac{2}{n+1}} & \text { for } n \geq 4 .\end{cases}
$$

The case $n=2$, settled by Bonnesen, has been included here for comparison.

The estimate (II) is sharp also in dimension $n \geq 3$, except for the value of $C$. To see this we construct (in §3) an explicit one-parameter family of convex bodies $D=D_{\alpha}, \alpha \in \mathbf{R}_{+}$, such that, for each $D_{\alpha}$, (II) holds with $\leq$ replaced by $\geq$, but with $C$ replaced by a suitable smaller constant independent of $\alpha$; and further such that the isoperimetric deficiency of $D_{\alpha}$ approaches 0 as $\alpha \rightarrow 0$.

In order to derive these precise estimates (II) from (I.b) we first note the following elementary geometric inequality, valid for any convex body $D$,

$$
\|\nabla u\|_{\infty} \leq 2 \frac{1+\|u\|_{\infty}}{1-\|u\|_{\infty}}\|u\|_{\infty}^{\frac{1}{2}}
$$

(Lemma 2.2). It shows that the former condition in (*) implies the latter (when $n \geq 3$ ), hence further implies (I), which in turn easily leads to (II) when $\Delta$ is sufficiently small, again in view of $(* *)$. To complete the proof we consider, for any convex body $D$, the family of parallel bodies $D(\lambda)=D+\lambda \Omega(\Omega=$ the closed unit ball, $\lambda \geq 0)$. Using a classical inequality of Minkowski it is easily shown that the isoperimetric deficiency $\Delta(\lambda)$ of $D(\lambda)$ decreases to 0 as $\lambda \rightarrow \infty$ (Lemma 2.5). On the other hand, the barycentre of $D(\lambda)$ remains bounded, 
and hence the "spherical deviation" $\|u\|_{\infty}$, now calculated for $D(\lambda)$, tends to 0 as $\lambda \rightarrow \infty$, showing that (II) holds when applied to $D(\lambda)$ for all sufficiently large $\lambda$. A simple continuity argument finishes the proof.

In a preliminary version of the present paper the stability result (II) for convex bodies satisfying $\Delta<\eta$ was established for $n=3$ (with a much smaller value of $\eta$ ) by use of two inequalities due to Bonnesen [3, p. 135], and for general $n \geq 3$ by application of a recent result of Osserman [10] (cf. $\S 2.7$ below) combined with the following estimate

$$
\frac{\delta}{\rho} \leq 2^{n} n^{2}\left(\frac{n}{n-1}\right)^{n-1}(1+\Delta)^{n}
$$

of the ratio between the diameter $\delta$ and the inradius $\rho$ of a convex body in $\mathbf{R}^{n}$ in terms of the isoperimetric deficiency $\Delta$.

\section{STABILITY IN THE CASE OF NEARLY SPHERICAL DOMAINS}

The isoperimetric deficiency of a (compact) domain $D$ in $\mathbf{R}^{n}$ of $n$-dimensional volume $V$ and $(n-1)$-dimensional surface area $S$ is defined in this paper by

$$
\Delta=\frac{S}{n \omega_{n}}\left(\frac{V}{\omega_{n}}\right)^{-\frac{n-1}{n}}-1
$$

where $\omega_{n}$ denotes the volume of the unit ball $\Omega$ in $\mathbf{R}^{n}$. The isoperimetric theorem asserts that $\Delta \geq 0$, with equality only for a ball.

The barycentre of $D$ is denoted by

$$
b=\frac{1}{V} \int_{D} x d x
$$

where $d x$ refers to Lebesgue measure on $\mathbf{R}^{n}$. We say that $D$ is normalized if $V=\omega_{n}$ and $b=0$. Any domain can be normalized, i.e., translated and transformed homothetically into the normalized domain $v^{-1}(D-b)$, where the volume radius $v>0$ is defined by $V=\omega_{n} v^{n}$.

We shall always assume that $D$ is compact and furthermore starshaped with respect to its barycentre $b$, in the strong sense that $b$ is an inner point of $D$ and that each ray issuing from $b$ meets the boundary $\partial D$ in exactly one point. After normalization the boundary is therefore representable in polar coordinates $(R, \xi)$ by an equation

$$
R=R(\xi)=1+u(\xi), \quad \xi \in \Sigma,
$$

where $\Sigma$ denotes the unit sphere in $\mathbf{R}^{n}$ and $R=|x|$ the Euclidean norm of $x \in \mathbf{R}^{n}$.

Let $\sigma$ denote the normalized surface measure on $\Sigma$. For any $p, 1 \leq p \leq \infty$, $\|\cdot\|_{p}$ denotes the usual norm on $L^{p}(\sigma)$. In particular, for a Lipschitz function $u: \Sigma \rightarrow \mathbf{R}:$

$$
\|u\|_{\infty}=\max _{\xi \in \Sigma}|u(\xi)|, \quad\|\nabla u\|_{\infty}=\underset{\xi \in \Sigma}{\operatorname{ess} \sup }|\nabla u(\xi)|,
$$


where $\nabla u$ denotes the gradient of $u$ (defined $\sigma$-almost everywhere on $\Sigma$ ). Of course, $\|\nabla u\|_{p}$ means $\||\nabla u|\|_{p}$.

1.1. Definition. A domain $D$ in $\mathbf{R}^{n}$ will be called nearly spherical if $D$, after normalization, has its boundary represented as in (2) with $u(\cdot)$ of Lipschitz class and satisfying

$$
\|u\|_{\infty} \leq a:=\frac{3}{20 n}, \quad\|\nabla u\|_{\infty} \leq \frac{1}{2} .
$$

Note that $a \leq 1 / 20$ for $n \geq 3$. For a convex domain, the latter inequality in (3) is therefore a consequence of the former (when $n \geq 3$ ), see Lemma 2.2 below. (The above constants $a$ and $1 / 2$ have been chosen primarily so as to ensure this implication and make the proof of the following theorem work.)

1.2. Theorem. For any nearly spherical domain $D$ in $\mathbf{R}^{n}$ we have

$$
\begin{gathered}
\frac{1}{10}\left(\|u\|_{2}^{2}+\|\nabla u\|_{2}^{2}\right) \leq \Delta \leq \frac{3}{5}\|\nabla u\|_{2}^{2}, \\
\|u\|_{\infty}^{n-1} \leq \begin{cases}A \Delta^{\frac{1}{2}} & \text { for } n=2, \\
A \Delta \log \frac{A^{\prime}\|\nabla u\|_{\infty}^{2}}{\Delta} & \text { for } n=3, \Delta>0, \\
A \Delta\|\nabla u\|_{\infty}^{n-3} & \text { for } n \geq 4,\end{cases}
\end{gathered}
$$

where $A$ and $A^{\prime}$ only depend on $n$ and are explicitly calculable.

Note that, in (5), one may replace $\|\nabla u\|_{\infty}$ by $1 / 2$ in view of (3). Explicit values of $A, A^{\prime}$ are given in Remark 1.5.

Proof. We may suppose that $D$ is normalized from the outset; hence, by (1),

$$
\Delta=\frac{S}{n \omega_{n}}-1 \text {. }
$$

The surface area $S$, the volume $V\left(=\omega_{n}\right)$, and the barycentre $b(=0)$ of $D$ are then given by

$$
\begin{aligned}
\frac{S}{n \omega_{n}} & =\int_{\Sigma} R^{n-2} \sqrt{R^{2}+|\nabla R|^{2}} d \sigma \\
\frac{S}{n \omega_{n}} & =\int_{\Sigma}(1+u)^{n-1} \sqrt{1+(1+u)^{-2}|\nabla u|^{2}} d \sigma \\
\frac{V}{\omega_{n}} & =\int_{\Sigma}(1+u)^{n} d \sigma \quad\left(=\int_{\Sigma} 1 d \sigma=1\right) \\
b & =\int_{\Sigma}(1+u(\xi))^{n+1} \xi d \sigma(\xi) \quad(=0) .
\end{aligned}
$$

In order to exploit (7) we introduce the following function $v$ close to $u$ :

$$
v=\frac{1}{n}\left((1+u)^{n}-1\right), \quad u=(1+n v)^{\frac{1}{n}}-1,
$$


whereby (7) reads

$$
\int_{\Sigma} v d \sigma=0
$$

In the sequel we occasionally assume that $n \geq 3$, but the planar case can be handled in the same way, cf. Remark 1.5 below.

We estimate $|v-u|$ and $|\nabla v-\nabla u|$, using (3):

$$
|v-u|=\frac{1}{n}\left|\sum_{k=2}^{n}\left(\begin{array}{l}
n \\
k
\end{array}\right) u^{k}\right| \leq \frac{1}{n}\left(\begin{array}{l}
n \\
2
\end{array}\right) a|u| \sum_{k=2}^{\infty}\left(\frac{n-2}{3} a\right)^{k-2}=\frac{3(n-1) a}{6-2(n-2) a}|u|
$$

$$
\begin{gathered}
|v-u| \leq \frac{3}{38}|u|, \\
\frac{35}{38}|u| \leq|v| \leq \frac{41}{38}|u| . \\
|\nabla v-\nabla u|=\left|(1+u)^{n-1}-1\right||\nabla u| \leq \sum_{k=1}^{n-1}\left(\begin{array}{c}
n-1 \\
k
\end{array}\right) a^{k}|\nabla u| \\
\leq(n-1) a|\nabla u| \sum_{k=1}^{\infty}\left(\frac{n-2}{2} a\right)^{k-1}=\frac{2(n-1) a}{2-(n-2) a}|\nabla u|, \\
|\nabla v-\nabla u| \leq \frac{6}{37}|\nabla u|, \\
\frac{31}{37}|\nabla u| \leq|\nabla v| \leq \frac{43}{37}|\nabla u| .
\end{gathered}
$$

$1^{\circ}$. Estimate of $\Delta$ from below. The proof of the former inequality in (4) is rather delicate. When expanding the integral on the right of (6) it is important to eliminate the integral of the first order term in $(1+u)^{n-1}$, and this will be done by use of (7):

$$
\begin{aligned}
\int_{\Sigma}(1+u)^{n-1} d \sigma & =\int_{\Sigma}(1+u)^{n-1} d \sigma-\frac{n-1}{n} \int_{\Sigma}\left((1+u)^{n}-1\right) d \sigma \\
& =1+\sum_{k=2}^{n}\left[\left(\begin{array}{c}
n-1 \\
k
\end{array}\right)-\frac{n-1}{n}\left(\begin{array}{l}
n \\
k
\end{array}\right)\right] \int_{\Sigma} u^{k} d \sigma \\
& =1-\sum_{k=2}^{n} \frac{k-1}{k}\left(\begin{array}{l}
n-1 \\
k-1
\end{array}\right) \int_{\Sigma} u^{k} d \sigma, \\
\left|\int_{\Sigma}(1+u)^{n-1} d \sigma-1\right| & \leq(n-1)\left[\frac{1}{2}+\sum_{k=3}^{\infty}\left(\frac{n-2}{2} a\right)^{k-2}\right] \int_{\Sigma} u^{2} d \sigma .
\end{aligned}
$$

Writing, for simplicity, $\|\cdot\|$ in place of $\|\cdot\|_{2}$ in the rest of the proof, we infer that

$$
1 \geq \int_{\Sigma}(1+u)^{n-1} d \sigma \geq 1-(n-1) \beta\|u\|^{2}, \quad \beta=\frac{43}{74}
$$


Because $\sqrt{1+t} \geq 1+\frac{t}{2}\left(1-\frac{t}{4}\right)$ for $t \geq 0$, and $|\nabla u|^{2} \leq \frac{1}{4}$, cf. (3), we have

$$
\begin{aligned}
&(1+u)^{n-1} \sqrt{1+(1+u)^{-2}|\nabla u|^{2}} \\
& \geq(1+u)^{n-1}+\frac{1}{2}(1+u)^{n-3}|\nabla u|^{2}\left(1-\frac{1}{16}(1+u)^{-2}\right) .
\end{aligned}
$$

For $n \geq 3$ we insert

$$
(1+u)^{n-3} \geq 1-(n-3)|u| \geq 1-n a=\frac{17}{20}
$$

and $(1+u)^{-2} \leq\left(1-\frac{1}{20}\right)^{-2}$ in $(15)$, and we obtain after integration over $\Sigma$, invoking (6) and the latter inequality in (14),

$$
\Delta=\frac{S}{n \omega_{n}}-1 \geq-(n-1) \beta\|u\|^{2}+\gamma\|\nabla u\|^{2}
$$

with $\beta=\frac{43}{74}, \gamma=\frac{17}{40}\left(1-\left(\frac{5}{19}\right)^{2}\right)=\frac{714}{1805}$.

Denoting by $\nabla^{2}$ the Laplace-Beltrami operator on $\Sigma$, we have

$$
\|\nabla u\|^{2}=-\int_{\Sigma} u \nabla^{2} u d \sigma
$$

The projection of $u$ on the eigenspace of $-\nabla^{2}$ corresponding to the eigenvalue $k(k+n-2), k=0,1,2, \ldots$, may be written as $a_{k} Y_{k}$, where $a_{k} \geq 0$ is a constant and $Y_{k}$ is a normalized eigenfunction, hence the restriction to $\Sigma$ of a (real) homogeneous polynomium of degree $k$ on $\mathbf{R}^{n}$ (cf. e.g. [9]). We thus have the following expansion in spherical harmonics

$$
\begin{gathered}
u=\sum_{k=0}^{\infty} a_{k} Y_{k}, \quad-\nabla^{2} Y_{k}=k(k+n-2) Y_{k}, \\
\|u\|^{2}=\sum_{k=0}^{\infty} a_{k}^{2}, \quad\|\nabla u\|^{2}=\sum_{k=1}^{\infty} k(k+n-2) a_{k}^{2},
\end{gathered}
$$

in view of (17). viz.

From (7), (8) we shall now deduce that $a_{k}=\int_{\Sigma} u Y_{k} d \sigma$ is small for $k=0,1$,

$$
a_{0} \leq \alpha\|u\|, \quad a_{1} \leq \alpha\|u\|, \quad \alpha=\frac{3}{38} .
$$

As to $a_{0}$, note that $Y_{0}=1$ (or -1 ), and so from (10), (11)

$$
a_{0}=\left|\int_{\Sigma} u d \sigma\right|=\left|\int_{\Sigma}(u-v) d \sigma\right| \leq \int_{\Sigma}|u-v| d \sigma \leq \alpha \int_{\Sigma}|u| d \sigma \leq \alpha\|u\| .
$$

As to $a_{1}, Y_{1}$ is the restriction to $\Sigma$ of a linear form on $\mathbf{R}^{n}$, and hence, by (8) and by symmetry,

$$
\int_{\Sigma}(1+u)^{n+1} Y_{1} d \sigma=0, \quad \int_{\Sigma} Y_{1} d \sigma=0
$$


We therefore obtain, using $\left\|Y_{1}\right\|=1$,

$$
\begin{aligned}
a_{1} & =\frac{-1}{n+1} \int_{\Sigma}\left[(1+u)^{n+1}-1-(n+1) u\right] Y_{1} d \sigma \\
& =\frac{-1}{n+1} \sum_{k=2}^{n+1}\left(\begin{array}{c}
n+1 \\
k
\end{array}\right) \int_{\Sigma} u^{k} Y_{1} d \sigma \\
& \leq \frac{1}{n+1} \sum_{k=2}^{n+1}\left(\begin{array}{c}
n+1 \\
k
\end{array}\right)\left\|u^{k}\right\| \leq \frac{n a}{2} \sum_{k=2}^{\infty}\left(\frac{n-1}{3} a\right)^{k-2}\|u\| \leq \alpha\|u\|,
\end{aligned}
$$

again with $\alpha=\frac{3}{38}$, because $(n-1) a \leq \frac{3}{20}$ by (3).

From (19), (18) we find

$$
\begin{gathered}
a_{0}^{2}+a_{1}^{2} \leq 2 \alpha^{2} \sum_{k=0}^{\infty} a_{k}^{2}, \\
\|u\|^{2}=\sum_{k=0}^{\infty} a_{k}^{2} \leq \lambda \sum_{k=2}^{\infty} a_{k}^{2},
\end{gathered}
$$

where

$$
\lambda=\left(1-2 \alpha^{2}\right)^{-1}, \quad \alpha=\frac{3}{38} .
$$

We infer from (18), pulling out the terms corresponding to $k=0,1$, that

$$
\|u\|^{2}+\|\nabla u\|^{2}=a_{0}^{2}+n a_{1}^{2}+\sum_{k=2}^{\infty}[k(k+n-2)+1] a_{k}^{2},
$$

whence, in view of (19), (20),

$$
\|u\|^{2}+\|\nabla u\|^{2} \leq \sum_{k=2}^{\infty}\left[k(k+n-2)+1+(n+1) \alpha^{2} \lambda\right] a_{k}^{2} .
$$

Similarly, from (16),

$$
\Delta \geq-(n-1) \beta a_{0}^{2}-(n-1)(\beta-\gamma) a_{1}^{2}+\sum_{k=2}^{\infty}[\gamma k(k+n-2)-(n-1) \beta] a_{k}^{2},
$$

and here (19) and (20) are applicable because $\beta-\gamma>0$. This leads to

$$
\Delta \geq \sum_{k=2}^{\infty}\left[\gamma k(k+n-2)-(n-1)\left(\beta-\alpha^{2} \gamma\right) \lambda\right] a_{k}^{2}
$$

noting that $1+2 \alpha^{2} \lambda=\lambda$ by $(21)$.

Now multiply (22) by a positive constant $\kappa_{n}<\gamma$ to be specified presently, and subtract from (23):

$$
\begin{aligned}
\Delta & -\kappa_{n}\left(\|u\|^{2}+\|\nabla u\|^{2}\right) \\
& \geq \sum_{k=2}^{\infty}\left[\left(\gamma-\kappa_{n}\right) k(k+n-2)-(n-1)\left(\beta-\alpha^{2} \gamma\right) \lambda-\kappa_{n}\left(1+(n+1) \alpha^{2} \lambda\right)\right] a_{k}^{2} .
\end{aligned}
$$


The coefficient to $a_{k}^{2}$ increases with $k$. To ensure that all coefficients are $\geq 0$ it suffices therefore to choose $\kappa_{n}$ so that the coefficient to $a_{2}^{2}$ equals 0 :

$$
\kappa_{n}=\frac{\left(2 \gamma-\left(\beta-\alpha^{2} \gamma\right) \lambda\right) n+\left(\beta-\alpha^{2} \gamma\right) \lambda}{\left(2+\alpha^{2} \lambda\right) n+\left(1+\alpha^{2} \lambda\right)} .
$$

Inserting $\alpha=\frac{3}{38}, \beta=\frac{43}{74}, \gamma=\frac{714}{1805}$, and $\lambda$ (see (16), (21)), we obtain, writing $\lim _{n \rightarrow \infty} \kappa_{n}=\kappa$,

$$
\kappa=0.102 \ldots>\frac{1}{10}, \quad \kappa_{3}=0.171 \ldots
$$

The monotone sequence $\left(\kappa_{n}\right)$ is thus decreasing and $\kappa_{n}>\frac{1}{10}$. Note also that $\kappa_{n} \leq \kappa_{3}<\gamma$. We have therefore now established the former inequality in (4) in the stronger form

$$
\Delta \geq \kappa_{n}\left(\|u\|^{2}+\|\nabla u\|^{2}\right) .
$$

$2^{\circ}$. Estimate of $\Delta$ from above. For the easy proof of the latter inequality in (4) we only need the assumption $|u| \leq a=3 /(20 n)$ in (3). Noting that $\sqrt{1+t} \leq 1+\frac{1}{2} t$ for $t \geq 0$, we get from (6)

$$
\frac{S}{n \omega_{n}} \leq \int_{\Sigma}\left[(1+u)^{n-1}+\frac{1}{2}(1+u)^{n-3}|\nabla u|^{2}\right] d \sigma
$$

with

$$
(1+u)^{n-3} \leq\left(1+\frac{3}{20 n}\right)^{n} \leq e^{0.15} .
$$

Invoking the former inequality in (14), we obtain from (24) the desired estimate

$$
\Delta=\frac{S}{n \omega_{n}}-1 \leq \frac{1}{2} e^{0.15}\|\nabla u\|^{2} \leq \frac{3}{5}\|\nabla u\|^{2} .
$$

$3^{\circ}$. Proof of (5). These estimates are immediate consequences of Lemma 1.4 below in view of (10), (12), (13), and (4).

1.3. Remark. The above theorem implies of course an estimate of $\|u\|_{2}$ in terms of $\|\nabla u\|_{2}$. A better estimate results from (20) and (18):

$$
\|u\|_{2}^{2} \leq \lambda \sum_{k=2}^{\infty} a_{k}^{2} \leq \frac{\lambda}{2 n} \sum_{k=2}^{\infty} k(k+n-2) a_{k}^{2} \leq \frac{\lambda}{2 n}\|\nabla u\|_{2}^{2} .
$$

1.4. Lemma. For any Lipschitz function $v$ on the unit sphere $\Sigma$ in $\mathbf{R}^{n}$ such that the mean value $\int_{\Sigma} v d \sigma$ equals 0 , we have

$$
\|v\|_{\infty}^{n-1} \leq \begin{cases}\pi\|\nabla v\|_{1} \leq \pi\|\nabla v\|_{2} & \text { for } n=2, \\ 4\|\nabla v\|_{2}^{2} \log \frac{8 e\|\nabla v\|_{\infty}^{2}}{\|\nabla v\|_{2}^{2}} & \text { for } n=3, v \neq 0, \\ B\|\nabla v\|_{2}^{2}\|\nabla v\|_{\infty}^{n-3} & \text { for } n \geq 4,\end{cases}
$$

where $B$ only depends on $n$ and is explicitly calculable. 
Proof. Fix a point $\xi^{*} \in \Sigma$ and use it as "north pole" for spherical coordinates $\varphi \in[0, \pi], \theta \in \Sigma^{*}$, where $\Sigma^{*}$ denotes the unit sphere in the hyperplane $\xi^{*} \cdot \xi=$ 0 . When $d \theta$ refers to the normalized surface measure on $\Sigma^{*}$ we thus have

$$
d \sigma=K^{-1} \sin ^{n-2} \varphi d \varphi d \theta,
$$

where

$$
K=\int_{0}^{\pi} \sin ^{n-2} \varphi d \varphi
$$

For any $\xi \in \Sigma \backslash\left\{\xi^{*},-\xi^{*}\right\}$ we have

$$
v(\xi)-v\left(\xi^{*}\right)=\int_{\xi^{*}}^{\xi} \nabla v(\eta) \cdot d \eta,
$$

integrated along the meridian through $\xi^{*}$ and $\xi$. Integration with respect to $d \sigma(\xi)$ leads to

$$
\left|v\left(\xi^{*}\right)-\int_{\Sigma} v d \sigma\right| \leq K^{-1} \int_{\Sigma^{*}} d \theta \int_{0}^{\pi} \sin ^{n-2} \varphi d \varphi \int_{0}^{\varphi}|\nabla v(\psi, \theta)| d \psi,
$$

where of course $\nabla v(\psi, \theta)$ means the value of $\nabla v$ at the point of $\Sigma$ with spherical coordinates $(\psi, \theta)$. In the integral with respect to $\psi$ we replace $\varphi$ by $\pi$ and thus obtain, invoking (25) and the definition of $K$, and inserting $\int_{\Sigma} v d \sigma=0$,

$$
\left|v\left(\xi^{*}\right)\right| \leq \int_{\Sigma^{*}} d \theta \int_{0}^{\pi}|\nabla v(\psi, \theta)| d \psi=K \int_{\Sigma} \sin ^{2-n} \varphi|\nabla v(\xi)| d \sigma(\xi) .
$$

For $n=2$ this leads immediately to the stated inequalities because $K=\pi$ here.

For $n \geq 3$ we estimate $|\nabla v(\xi)|$ by $\|\nabla v\|_{\infty}$ on the two caps of $\Sigma$ given by $\sin \varphi \leq \sin \alpha$, for some $\alpha \in] 0, \pi / 2[$ to be chosen presently. On the rest of $\Sigma$ we use the Cauchy-Schwarz inequality, and we thus obtain

$$
\begin{aligned}
\left|v\left(\xi^{*}\right)\right| \leq & \|\nabla v\|_{\infty} K \int_{\sin \varphi \leq \sin \alpha} \sin ^{2-n} \varphi d \sigma(\xi) \\
& +K\left(\int_{\sin \varphi>\sin \alpha} \sin ^{4-2 n} \varphi d \sigma(\xi)\right)^{\frac{1}{2}}\|\nabla v\|_{2} .
\end{aligned}
$$

Inserting (25), and maximizing over $\xi^{*} \in \Sigma$, leads to

$$
\|v\|_{\infty} \leq 2 \alpha\|\nabla v\|_{\infty}+\left(2 K \int_{\alpha}^{\pi / 2} \sin ^{2-n} \varphi d \varphi\right)^{\frac{1}{2}}\|\nabla v\|_{2} .
$$

$1^{\circ}$. The case $n=3$. Here $K=2$, and we get from (26)

$$
\|v\|_{\infty} \leq 2 \alpha\|\nabla v\|_{\infty}+2\left(\log \cot \frac{\alpha}{2}\right)^{\frac{1}{2}}\|\nabla v\|_{2} .
$$

Because $\cot \frac{\alpha}{2} \leq \frac{2}{\alpha}$ and $(a+b)^{2} \leq 2 a^{2}+2 b^{2}$, this leads to the more manageable inequality

$$
\|v\|_{\infty}^{2} \leq 8 \alpha^{2}\|\nabla v\|_{\infty}^{2}+8 \log \frac{2}{\alpha}\|\nabla v\|_{2}^{2}
$$


From $v \neq 0$ and $\int v d \sigma=0$ follows $\|\nabla v\|_{2}>0$, and the right-hand member in (27) is least for $2 \alpha^{2}=\|\nabla v\|_{2}^{2} /\|\nabla v\|_{\infty}^{2}$. Inserting this in (27) we obtain the estimates stated in the lemma for $n=3$.

$2^{\circ}$. The case $n \geq 4$. Because $\sin \varphi \geq 2 \varphi / \pi$, we now get from (26)

$$
\|v\|_{\infty} \leq 2 \alpha\|\nabla v\|_{\infty}+(2 K)^{\frac{1}{2}}\left(\frac{\pi}{2}\right)^{\frac{n-2}{2}} \frac{\alpha^{\frac{3-n}{2}}}{\sqrt{n-3}}\|\nabla v\|_{2} .
$$

The right-hand member is least for $\alpha^{n-1}=K \frac{n-3}{8}\left(\frac{\pi}{2}\right)^{n-2}\|\nabla v\|_{2}^{2} /\|\nabla v\|_{\infty}^{2}$ (assuming $v \neq 0$, i.e., $\left.\|\nabla v\|_{2}>0\right)$. This leads, in the first place, to

$$
\|v\|_{\infty} \leq 2 \alpha \frac{n-1}{n-3}\|\nabla v\|_{\infty}
$$

and finally to the desired estimate with

$$
B=K \frac{n-1}{4}\left(\pi \frac{n-1}{n-3}\right)^{n-2} \text {. }
$$

1.5. Remark. For any specified dimension $n$ the estimates (4) and hence (5) in Theorem 1.2 can be readily improved, mainly by diminishing $\alpha, \beta, \gamma$ in the proof of (4). In this way the constants $\frac{1}{10}$ and $\frac{3}{5}$ in (4) can be replaced by 0.24 and 0.54, respectively, for $n=2$ and by $\frac{1}{4}$ and $\frac{1}{2}$, respectively, for $n=3$. If one cancels the term $\|u\|_{2}^{2}$ in (4), the constant $\frac{1}{10}$ in (4) may even be replaced by 0.30 for $n=2$ and by 0.29 for $n=3$. This leads to the following constants in (5) (when log denotes natural logarithm):

$$
\begin{array}{lll}
A=6.4 & \text { for } n=2, \\
A=18.5, \quad A^{\prime}=16.5 & \text { for } n=3 .
\end{array}
$$

With $B$ from the end of the proof of Lemma 1.4 we may clearly take

$$
A=10 B\left(\frac{38}{35} \cdot \frac{43}{37}\right)^{n-1} \quad \text { for } n \geq 4 .
$$

\section{Stability in the CASE OF CONVEX bodies}

Henceforth, $D$ denotes a convex body (= a compact convex set with nonempty interior) in $\mathbf{R}^{n}, n \geq 3 .{ }^{4}$ We denote by $v$, resp. $s$, the radius of a ball with the same volume $V$, resp. surface area $S$, as $D$ :

$$
V=\omega_{n} v^{n}, \quad S=n \omega_{n} s^{n-1} .
$$

The isoperimetric deficiency as defined in (1), $\S 1$, takes the form

$$
\Delta=\left(\frac{s}{v}\right)^{n-1}-1 \text {. }
$$

As before we denote by $b$ the barycentre of $D$; it is an inner point of $D$. The convex body $v^{-1}(D-b)$ has volume $\omega_{n}$ and barycentre 0 and is said to be normalized.

${ }^{4}$ In the case $n=2$ the function $f$ in Theorem 2.3 below would have the form $f(t)=C \sqrt{t}$, but the assertion of the theorem in this case is contained in Bonnesen's inequality quoted in the introduction. 
2.1. Definition. The spherical deviation of $D$ is defined by

$$
d=\min \left\{\alpha \geq 0 \mid(1-\alpha)_{+} \Omega \subset v^{-1}(D-b) \subset(1+\alpha) \Omega\right\} .
$$

Here $\Omega=\left\{x \in \mathbf{R}^{n}|| x \mid \leq 1\right\}$, and $(1-\alpha)_{+}=\max \{0,1-\alpha\}$.

The boundary of the normalized convex body $v^{-1}(D-b)$ is representable in polar coordinates in the form $\xi \mapsto R(\xi)=1+u(\xi), \xi \in \Sigma=\partial \Omega$, as in $\S 1$. Moreover, the function $u$ is Lipschitzian. Clearly,

$$
\|u\|_{\infty}=d
$$

2.2. Lemma. With the above notation we have

$$
\|\nabla u\|_{\infty} \leq 2 d^{1 / 2} \frac{1+d}{1-d}
$$

In particular, if $d \leq 1 / 20$ then

$$
\|\nabla u\|_{\infty} \leq \frac{42}{19} d^{1 / 2}<\frac{1}{2}
$$

Proof. Arguing like in the proof of a similar elementary inequality in [10] we find for almost every $\xi \in \Sigma$

$$
\frac{|\nabla u(\xi)|}{1+u(\xi)} \leq \tan \theta=\frac{2 \tan (\theta / 2)}{1-\tan ^{2}(\theta / 2)},
$$

where the angle $\theta$ is defined by

$$
\cos \theta=\frac{1-d}{1+d}, \quad \text { i.e. }, \quad \tan ^{2} \frac{\theta}{2}=d,
$$

whence the stated inequality because $1+u(\xi) \leq 1+d$.

Suppose for a moment that

$$
d \leq a:=\frac{3}{20 n} \quad\left(\leq \frac{1}{20}\right) .
$$

Then (30) shows that $D$ satisfies (3), $\S 1$; and hence (5) in Theorem 1.2 applies. For $n=3$ this means that (if $\Delta>0$ )

$$
d \leq c(\Delta \log (k / \Delta))^{\frac{1}{2}}
$$

with $c=A^{1 / 2}, k=A^{\prime} / 4$ (after inserting $\|\nabla u\|_{\infty} \leq 1 / 2$ ). For $n \geq 4$ we insert instead the former inequality (30) in (5) and obtain

$$
d \leq c \Delta^{\frac{2}{n+1}}
$$

where $c$ is determined by

$$
c^{\frac{n+1}{2}}=A \cdot\left(\frac{42}{19}\right)^{n-3}, \quad n \geq 4 \text {. }
$$

Summing up, we have found that

$$
d \leq a\left(=\frac{3}{20 n}\right) \quad \text { implies } \quad d \leq f(\Delta),
$$


where

$$
f(t)= \begin{cases}c(t \log (k / t))^{\frac{1}{2}} & \text { for } n=3 \\ c t^{\frac{2}{n+1}} & \text { for } n \geq 4,\end{cases}
$$

with $c$ and $k$ as specified above. (We define $f(0)=0$ if $n=3$.)

For $n \geq 4$ the continuous function $f$ is a strictly increasing self-mapping of $[0, \infty[$. For $n=3$ we shall consider $f$ as defined only on the interval $[0, k / e]$; then $f$ is strictly increasing and ranges from 0 to $c(k / e)^{1 / 2}(>5)$.

Now define the constant $\eta>0$, depending only on $n$, by

$$
f(\eta)=a \text {. }
$$

For $n=3$ this is possible because $a=1 / 20<5<f(k / e)$, and we find $\eta=1.04 \ldots \cdot 10^{-5}$. It remains to use the estimate

$$
\log \frac{k}{t} \leq c^{\prime} \log \frac{1}{t} \text { for } 0<t \leq \eta
$$

with $c^{\prime}=\log (k / \eta) / \log (1 / \eta)=1.123 \ldots$, whence

$$
f(t) \leq C\left(t \log \frac{1}{t}\right)^{1 / 2} \quad \text { for } 0<t \leq \eta, n=3,
$$

where we may take $C=c\left(c^{\prime}\right)^{1 / 2}=\left(A c^{\prime}\right)^{1 / 2}=4.55 \ldots$, cf. Remark 1.5.

We may now formulate our main result with reference to (32), (33), and (34) above.

2.3. Theorem. Every convex body $D$ in $\mathbf{R}^{n}$ of isoperimetric deficiency $\Delta<\eta$ has spherical deviation $d \leq f(\Delta)$. Explicitly we have (when $\Delta<\eta$ )

$$
d=\|u\|_{\infty} \leq \begin{cases}C\left(\Delta \log \frac{1}{\Delta}\right)^{\frac{1}{2}} & \text { for } n=3, \\ C \Delta^{\frac{2}{n+1}} & \text { for } n \geq 4,\end{cases}
$$

where the constant $C$ depends only on $n$ and is explicitly calculable.

It is understood that $\Delta \log (1 / \Delta)=0$ for $\Delta=0$.

For $n=3$ one may take $C=4.56, \eta=10^{-5}$. For $n \geq 4$ one may take $C=c=\left(\frac{42}{19}\right)^{\frac{2 n-6}{n+1}} A^{\frac{2}{n+1}}, \eta=\left(\frac{19}{42}\right)^{n-3}\left(\frac{3}{20 n}\right)^{\frac{n+1}{2}} A^{-1}$ (with $A$ from Theorem 1.2, cf . Remark 1.5).

For the proof of Theorem 2.3 we prepare the following two lemmas.

2.4. Lemma. The barycentre $b=b(D)$ and the spherical deviation $d=d(D)$ of a convex body $D$ are locally Lipschitzian (in particular continuous) functions of $D$ in the Hausdorff metric.

Proof. For any bounded measurable set $E \subset \mathbf{R}^{n}$ we write

$$
V(E)=\int_{E} 1 d x, \quad M(E)=\int_{E} x d x, \quad|E|=\sup \{|x| \mid x \in E\} .
$$


For two convex bodies $D, E$ (contained in the half-space $x_{1}>0$, for convenience), we may estimate the difference between the first coordinates $M_{1}(D)$, $M_{1}(E)$ of $M(D), M(E)$ in terms of the Hausdorff distance $\delta=\delta(D, E)$ (cf. $[4$, p. 34]) as follows

$$
\begin{aligned}
M_{1}(E) & \leq M_{1}(D)+M_{1}(E \backslash D), \\
M_{1}(E)-M_{1}(D) & \leq|E| V(E \backslash D) \\
& \leq(|D|+\delta)[V(D+\delta \Omega)-V(D)] \\
& =O(\delta) \text { as } \delta \rightarrow 0,
\end{aligned}
$$

uniformly for $D$ contained in a fixed ball. Here we use the fact that $V(D+\delta \Omega)-V(D)$ is a polynomial in $\delta$ taking the value 0 at 0 and having continuous coefficients (as functions of $D$ ), cf. e.g. [4, p. 40]. Similarly as to $M_{1}(D)-M_{1}(E)$, and consequently

$$
\left|M_{1}(E)-M_{1}(D)\right|=O(\delta) \quad \text { as } \delta \rightarrow 0,
$$

uniformly for $D$ contained in a fixed ball. In the same uniform sense we have $|V(E)-V(D)|=O(\delta)$. Having thus established that $M(D)$ and $V(D)$ are locally Lipschitzian as functions of $D$, we infer the same for the barycentre $b(D)=V(D)^{-1} M(D)$ because $V(D)>0$ for every convex body $D$. Finally, the spherical deviation $d(D)=d\left(D_{1}\right)$ is nothing but the Hausdorff distance $\delta\left(\Omega, D_{1}\right)$ between the unit ball $\Omega$ and the normalized body

$$
D_{1}=v(D)^{-1}(D-b(D)) \text {. }
$$

It is therefore likewise a locally Lipschitzian function of $D$.

2.5. Lemma. For any convex body $D$ in $\mathbf{R}^{n}$ the isoperimetric deficiency $\Delta(\lambda)$ of the parallel body $D(\lambda)=D+\lambda \Omega, \lambda \geq 0$, is a decreasing function of $\lambda$ and converges to 0 as $\lambda \rightarrow \infty$.

Proof. It is well known, see e.g. [4], that the volume $V(\lambda)$ and the surface area $S(\lambda)$ of $D(\lambda)$ are polynomials in $\lambda$ with nonnegative coefficients and of degree $n$ and $n-1$, respectively:

$$
V(\lambda)=\sum_{\nu=0}^{n}\left(\begin{array}{l}
n \\
\nu
\end{array}\right) W_{\nu} \lambda^{\nu}, \quad S(\lambda)=\sum_{\nu=1}^{n}\left(\begin{array}{l}
n \\
\nu
\end{array}\right) \nu W_{\nu} \lambda^{\nu-1}=V^{\prime}(\lambda) .
$$

In particular,

$$
W_{0}=V(0)=V, \quad n W_{1}=S(0)=S, \quad W_{n}=V(\Omega)=\omega_{n} .
$$

The logarithmic derivative of $n^{n} \omega_{n}(1+\Delta(\lambda))^{n}=S(\lambda)^{n} V(\lambda)^{1-n}$ at $\lambda=0$ is

$$
n \frac{S^{\prime}(0)}{S(0)}-(n-1) \frac{V^{\prime}(0)}{V(0)}=n(n-1) \frac{W_{0} W_{2}-W_{1}^{2}}{W_{0} W_{1}} \leq 0
$$

because $W_{1}^{2} \geq W_{0} W_{2}$, one of Minkowski's inequalities, cf. [4, p. 92]. Having thus found that $\Delta^{\prime}(0) \leq 0$ we infer that $\Delta^{\prime}(\lambda) \leq 0$ for any $\lambda \geq 0$, noting that, 
for $\mu \geq 0, \Delta(\lambda+\mu)$ is the isoperimetric deficiency of $D(\lambda+\mu)=(D(\lambda))(\mu)$. A similar easy calculation shows that $\Delta(\lambda)=O\left(1 / \lambda^{2}\right)$ as $\lambda \rightarrow \infty$, and more precisely

$$
\Delta(\lambda)=\frac{1}{2}(n-1)\left(W_{n-1}^{2}-W_{n} W_{n-2}\right) \omega_{n}^{-2} \lambda^{-2}+O\left(\lambda^{-3}\right) .
$$

Proof of Theorem 2.3. We may assume that $D$ is normalized: $v=1$ and $b=0$, and so

$$
(1-d)_{+} \Omega \subset D \subset(1+d) \Omega,
$$

by Definition 2.1. Theorem 2.3 will follow from Theorem 1.2 once it has been proved that

$$
\Delta<\eta \text { implies } d<a,
$$

cf. (31), (32), and (34).

To establish (39), consider again the parallel bodies $D(\lambda)=D+\lambda \Omega$. Write again $\Delta(\lambda)$ for the isoperimetric deficiency of $D(\lambda)$; and let $d(\lambda), b(\lambda)$, and $v(\lambda)$ be understood similarly. For $\lambda \geq(d-1)_{+}$we then have $1-d+\lambda \geq 0$ and $^{5}$

$$
\begin{gathered}
(1-d+\lambda) \Omega \subset D(\lambda) \subset(1+d+\lambda) \Omega, \\
\left(1-\frac{d}{1+\lambda}\right) \Omega \subset \frac{1}{1+\lambda} D(\lambda) \subset\left(1+\frac{d}{1+\lambda}\right) \Omega .
\end{gathered}
$$

It follows that the Hausdorff distance between $\Omega$ and $(1+\lambda)^{-1} D(\lambda)$ is $\leq$ $(1+\lambda)^{-1} d$. Applying Lemma 2.4 to the barycentres 0 and $(1+\lambda)^{-1} b(\lambda)$ of $\Omega$ and $(1+\lambda)^{-1} D(\lambda)$, respectively, we therefore obtain

$$
\frac{|b(\lambda)|}{1+\lambda}=O\left(\frac{d}{1+\lambda}\right)
$$

showing that $b(\lambda)$ remains bounded as $\lambda \rightarrow \infty$. From (40) we deduce

$$
(1+\lambda-d-|b(\lambda)|) \Omega \subset D(\lambda)-b(\lambda) \subset(1+\lambda+d+|b(\lambda)|) \Omega
$$

for $\lambda$ large enough so that $1+\lambda>d+|b(\lambda)|$. From (38) together with $W_{n}=\omega_{n}$ we obtain $v(\lambda)=\lambda+O(1)$. Dividing by $v(\lambda)$ in (41) we therefore find that the normalized body $v(\lambda)^{-1}(D(\lambda)-b(\lambda))$ is squeezed between two balls centered at 0 and of radii which both have the form $1+O(1 / \lambda)$. By Definition 2.1 this means that

$$
d(\lambda)=O(1 / \lambda) \text { as } \lambda \rightarrow \infty .
$$

Now suppose that $\Delta<\eta$ and, by contradiction, that $d \geq a$, cf. (39). Because $d(\lambda)$ is continuous in $\lambda$ (Lemma 2.4) and tends to 0 as $\lambda \rightarrow \infty$, there exists $\lambda \geq 0$ such that $d(\lambda)=a$. By Lemma 2.5, $\Delta(\lambda) \leq \Delta<\eta$. Applying (31) to $D(\lambda)$ and recalling that $f$ is strictly increasing, cf. (32) and subsequent lines, we are led to the contradiction

$$
a=d(\lambda) \leq f(\Delta(\lambda))<f(\eta)=a,
$$

${ }^{5}$ If $d \geq 1$ the former relation (40) follows from $0 \leq 1-d+\lambda \leq \lambda$, noting that $0 \in D$ and so $\lambda \Omega \subset D(\lambda)$. 
cf. (33). Consequently we actually have $d<a$, and the proof is complete.

2.7. Relation to a result by Osserman. Let $r$ and $\rho$ denote the circumradius and the inradius of a convex body $D$ in $\mathbf{R}^{n}, n \geq 3$. It follows easily from Lemma 2.4 that the spherical deviation $d$ of $D$ is equivalent to $(r-\rho) / \rho$ for small values of either quantity, in the sense that $(r-\rho) /(\rho d)$ is bounded and bounded away from 0 for such small values. Hence Theorem 2.3 implies a recent result by Osserman [10] according to which there exist explicitly calculable positive constants $c, \gamma$ depending only on $n$ such that

$$
\Delta_{1}<\gamma \quad \text { implies } \frac{r-\rho}{\rho} \leq c \Delta_{1}^{k}, \quad k=\frac{2}{n(n+1)} .
$$

Here $\Delta_{1}$ denotes the following version of the isoperimetric deficiency involving the inradius $\rho$ :

$$
\Delta_{1}=\frac{s^{n}-v^{n}}{\rho^{n}}=\left[\left(\frac{s}{v}\right)^{n}-1\right]\left(\frac{v}{\rho}\right)^{n},
$$

and (42) is therefore not a stability result in our sense. We have

$$
\Delta \leq \Delta_{1} \leq \frac{n}{n-1} \Delta(1+\Delta)^{\frac{1}{n-1}}\left(\frac{v}{\rho}\right)^{n} .
$$

The former inequality follows from $\rho \leq v \leq s$, and the latter is obtained by applying the mean value theorem to $(s / v)^{n}-1=(1+\Delta)^{n / n-1}-1$.

Actually, the stronger version of (42) in which $\Delta_{1}$ is replaced by $\Delta\left(\leq \Delta_{1}\right)$ is an immediate consequence of Theorem 2.3, because the function $t^{k}$ with $k$ as stated in (42) is large (for small $t$ ) compared to our $(t \log (1 / t))^{1 / 2}$ and $t^{2 /(n+1)}$ for $n=3$ and $n \geq 4$, respectively.

Theorem 2.3 further implies that $\Delta$ and $\Delta_{1}$ are in fact equivalent for small values of either quantity. Since $\Delta \leq \Delta_{1}$, this follows from $\rho \geq(1-d) v>$ $(1-a) v$ for $\Delta<\eta$, cf. (39), whence $v / \rho$ in (43) remains bounded for $\Delta<\eta$.

Osserman's proof of $(42)$ is based on the inequality (for convex bodies $D$ )

$$
\left(\frac{s}{\rho}-1\right)^{n} \leq \Delta_{1}
$$

due to Bonnesen [3, p. 63] for $n=2$, to Hadwiger [7] for $n=3$, and to Dinghas [5] (in a sharpened form) for general $n$. To derive a stability result (quantitatively much weaker than Theorem 2.3) directly from Osserman's result (42) it suffices to note that $v \leq \delta / 2(\delta=$ the diameter of $D)$ and to apply the nontrivial estimate of $\delta / \rho$ stated at the end of the introduction.

\section{EXAMPLES SHOWING THAT THE RESUltS ARE SHARP}

The estimates in Theorems 1.2 and 2.3, and in Lemma 1.4, are sharp as far as the orders of magnitude as $\Delta \rightarrow 0$ are concerned. For $n=2$ this follows from Bonnesen's theorem [2]. For $n \geq 3$ we shall construct two examples (one for $n=3$ and the other for $n \geq 4$ ) of a one-parameter family of convex bodies $\left(D_{c}\right)_{a>0}$ in $\mathbf{R}^{n}$ such that $\|u\|_{\infty}$ (in Theorem 1.2), $\|v\|_{\infty}$ (in Lemma 1.4), and 
the spherical deviation $d=\|u\|_{\infty}$ (in Theorem 2.3), all evaluated for $D_{\alpha}$, have precisely the stated order of magnitude in their dependence on the isoperimetric deficiency $\Delta\left(D_{\alpha}\right)$ of $D_{\alpha}$, and such that $\Delta\left(D_{\alpha}\right) \rightarrow 0$ as $\alpha \rightarrow 0$.

3.1. Example. $(n=3)$. This example arose upon inspection of the proof of Lemma 1.4. It can hardly be appreciably simplified. (In particular, the very simple type of example which serves in the case $n \geq 4$, cf. $\S 3.2$ below, would be far from giving the correct order of magnitude in the present, more subtle case $n=3$.)

For any $\alpha, 0<\alpha<\pi / 2$, consider the following function $w=w(\xi)$ on the unit sphere $\Sigma$ in $\mathbf{R}^{3}$, depending only on the spherical distance $\varphi, 0 \leq \varphi \leq \pi$, from a prescribed "north pole" $\xi^{*} \in \Sigma$, whence we may also write $w=w(\varphi)$ :

$$
\begin{gathered}
w= \begin{cases}-\sin ^{2} \alpha \log \sin \alpha+\sin \alpha(\sin \alpha-\sin \varphi) & \text { for } \sin \varphi \leq \sin \alpha, \\
-\sin ^{2} \alpha \log \sin \varphi & \text { for } \sin \varphi \geq \sin \alpha,\end{cases} \\
w^{\prime}= \begin{cases}-\sin \alpha \cos \varphi & \text { for } \sin \varphi \leq \sin \alpha, \\
-\sin ^{2} \alpha \cot \varphi & \text { for } \sin \varphi \geq \sin \alpha,\end{cases} \\
w^{\prime \prime}= \begin{cases}\sin \alpha \sin \varphi & \text { for } \sin \varphi<\sin \alpha, \\
\sin ^{2} \alpha / \sin ^{2} \varphi & \text { for } \sin \varphi>\sin \alpha .\end{cases}
\end{gathered}
$$

It is easily verified that $w$ is of class $C^{1}$. Clearly, $w(\pi-\varphi)=w(\varphi)$. Moreover, $w \geq 0$, and for $0 \leq \varphi \leq \pi / 2$ we have $w^{\prime}(\varphi) \leq 0$. The mean value of $w$ as a fuction on $\Sigma$ is

$$
\begin{aligned}
\bar{w} & =\int_{0}^{\pi / 2} w \sin \varphi d \varphi \\
& =\sin ^{2} \alpha\left(1+\log \frac{1}{\sin \alpha}-\log \cot \frac{\alpha}{2}-\frac{1}{2} \frac{\alpha}{\sin \alpha}+\frac{1}{2} \cos \alpha\right), \\
\bar{w} & =(1-\log 2) \alpha^{2}+O\left(\alpha^{3}\right) \quad \text { as } \alpha \rightarrow 0 .
\end{aligned}
$$

Now write

$$
\begin{gathered}
v=w-\bar{w}, \\
R=(1+3 v)^{\frac{1}{3}}=1+u,
\end{gathered}
$$

cf. (2), (9), §1. This $C^{1}$ function $R=R(\varphi), 0 \leq \varphi \leq \pi$, determines in planar polar coordinates $(R, \varphi)$ a convex curve because

$$
R^{2}+2\left(R^{\prime}\right)^{2}-R R^{\prime \prime} \geq 0 \quad \text { for } \sin \varphi \neq \sin \alpha,
$$

as we shall now see. From (45), (46) we get

$$
R^{2} R^{\prime}=v^{\prime}=w^{\prime}, \quad R^{2} R^{\prime \prime}+2 R\left(R^{\prime}\right)^{2}=w^{\prime \prime},
$$

and hence

$$
R\left(R^{2}+2\left(R^{\prime}\right)^{2}-R R^{\prime \prime}\right) \geq R^{3}-w^{\prime \prime}
$$


using that $2\left(R^{\prime}\right)^{2} \geq-2\left(R^{\prime}\right)^{2}$. It remains to show that $w^{\prime \prime} \leq R^{3}$. For $\sin \varphi \geq$ $2 \sin \alpha$ this follows from $w^{\prime \prime}=\sin ^{2} \alpha / \sin ^{2} \varphi \leq 1 / 4<R^{3}$ for small $\alpha$. In fact, because $w \geq 0, R^{3}=1+3 v \geq 1-3 \bar{w}=1-O\left(\alpha^{2}\right)$ by (44). Next, for $\sin \varphi \leq 2 \sin \alpha$, one finds $w^{\prime \prime} \leq 1$ (both for $\sin \varphi<\sin \alpha$ and for $\sin \varphi>\sin \alpha$ ), while $R \geq 1$ because $v \geq 0$; in fact, $w$ and hence $v$ are decreasing, and for $\sin \varphi=2 \sin \alpha$ the value of $v$ is

$$
\sin ^{2} \alpha \log \frac{1}{2 \sin \alpha}-\bar{w}=\alpha^{2}\left(\log \frac{1}{\alpha}-1\right)-O\left(\alpha^{3}\right)>0
$$

by (44), assuming again that $\alpha$ is sufficiently small.

Now view $v, u$, and $R$ from (45), (46) as $C^{1}$ functions on the unit sphere $\Sigma$. The equation $R=R(\xi)$ describes in polar coordinates the boundary of a convex body $D_{\alpha}$, obtained by rotating the above curve about the line $\mathbf{R} \xi^{*} . D_{\alpha}$ has barycentre at 0 , by symmetry, and volume

$$
V=\omega_{3} \int_{\Sigma} R^{3} d \sigma=\omega_{3} \int_{\Sigma}(1+3 v) d \sigma=\omega_{3},
$$

because the mean value of $v$ over $\Sigma$ is 0 . Thus $D_{\alpha}$ is normalized.

The maximum and minimum of $w(\varphi)$ are $w(0)=\sin ^{2} \alpha(1-\log \sin \alpha)$ and $w(\pi / 2)=0$, respectively. Because the mean value $\bar{w}$ is small compared to $w(0)$ (when $\alpha$ is small), we therefore have

$$
\|v\|_{\infty}=v(0)=w(0)-\bar{w}=\alpha^{2} \log \frac{1}{\alpha}+O\left(\alpha^{2}\right)
$$

by (44). Moreover,

$$
\|\nabla v\|_{\infty}=\|\nabla w\|_{\infty}=\max \left|w^{\prime}(\varphi)\right|=\left|w^{\prime}(0)\right|=\sin \alpha .
$$

In view of (46) it follows from (47) and (48) that also $\|u\|_{\infty}$ and $\|\nabla u\|_{\infty}$ approach 0 as $\alpha \rightarrow 0$. Hence $D_{\alpha}$ satisfies (3), $\S 1$, for small $\alpha$, and we infer from (47) together with (12), §1, that

$$
\|u\|_{\infty} \geq \frac{38}{41}\|v\|_{\infty}=\frac{38}{41} \alpha^{2} \log \frac{1}{\alpha}+O\left(\alpha^{2}\right) .
$$

Finally we estimate the isoperimetric deficiency $\Delta$ of $D_{\alpha}$, using (4) in Theorem 1.2. First,

$$
\begin{aligned}
\|\nabla v\|_{2}^{2} & =\|\nabla w\|_{2}^{2}=\int_{0}^{\pi / 2} w^{\prime}(\varphi)^{2} \sin \varphi d \varphi \\
& =\frac{1}{3} \sin ^{2} \alpha\left(1-\cos ^{3} \alpha\right)+\sin ^{4} \alpha\left(\log \cot \frac{\alpha}{2}-\cos \alpha\right) \\
& =\alpha^{4} \log \frac{1}{\alpha}+O\left(\alpha^{4}\right) .
\end{aligned}
$$

Combining this with (13) and (4), $\S 1$, we obtain

$$
\Delta \approx\|\nabla u\|_{2}^{2} \approx\|\nabla v\|_{2}^{2} \approx \alpha^{4} \log \frac{1}{\alpha},
$$


the sign $\approx$ between two functions of $\alpha$ meaning that their ratio in either order remains bounded for small $\alpha$. In particular, $\Delta \rightarrow 0$ as $\alpha \rightarrow 0$. The function $\alpha \log (1 / \alpha)$ being increasing for small $\alpha$, we infer that

$$
\Delta \log \frac{1}{\Delta}=O\left(\alpha^{4} \log \frac{1}{\alpha} \log \frac{1}{\alpha^{4} \log (1 / \alpha)}\right)=O\left(\alpha^{2} \log \frac{1}{\alpha}\right)^{2} .
$$

Inserting this in (47) and (49), we conclude that, for all sufficiently small $\alpha>0$, $\|u\|_{\infty}^{2}$ and $\|v\|_{\infty}^{2}$ do indeed exceed a positive constant times $\Delta \log (1 / \Delta)$.

3.2. Example. Let $\Omega$ denote the closed unit ball in $\mathbf{R}^{n}, n \geq 2$ (although only $n \geq 4$ is of interest). For a given $\alpha, 0<\alpha<\pi / 2$, let $D_{\alpha}$ denote the convex hull of $\Omega \cup\{p,-p\}$, where $p$ is a given point of $\mathbf{R}^{n}$ such that

$$
|p|=\frac{1}{\cos \alpha} \text {. }
$$

Thus $D_{\alpha}$ arises from $\Omega$ by adding two truncated cones of height $\sin ^{2} \alpha / \cos \alpha$ and of base radius $\sin \alpha$, from which cones are removed two caps of $\Omega$ of spherical radius $\alpha$. The volume of $D_{\alpha}$ is therefore

$$
\begin{aligned}
V & =\omega_{n}+\frac{2}{n} \omega_{n-1} \frac{\sin ^{n+1} \alpha}{\cos \alpha}-2 \omega_{n-1} \int_{0}^{\alpha} \sin ^{n} \varphi d \varphi \\
& =\omega_{n}+\frac{2}{n(n+1)} \omega_{n-1} \alpha^{n+1}+O\left(\alpha^{n+3}\right) .
\end{aligned}
$$

The surface of $D_{\alpha}$ is $S=n V$ because every tangent hyperplane to $\partial D_{\alpha}$ (except at the singular points $\pm p$ ) is at distance 1 from 0 . The volume radius of $D_{\alpha}$ is

$$
v=\left(\frac{V}{\omega_{n}}\right)^{1 / n}=1+\frac{2}{n^{2}(n+1)} \frac{\omega_{n-1}}{\omega_{n}} \alpha^{n+1}+O\left(\alpha^{n+3}\right) .
$$

The isoperimetric deficiency of $D_{\alpha}$ is

$$
\Delta=\frac{S}{n \omega_{n}}\left(\frac{V}{\omega_{n}}\right)^{-\frac{n-1}{n}}-1=v-1 \approx \alpha^{n+1} .
$$

We have

$$
\|\nabla u\|_{\infty}=|p| \tan \alpha \approx \alpha .
$$

The barycentre of $D_{\alpha}$ is 0 , by symmetry. The spherical deviation $d$ of $D_{\alpha}$ is between $v^{-1}\left(\frac{1}{\cos \alpha}-1\right)$ and half of that, and hence

$$
d \approx \alpha^{2} \approx \Delta^{\frac{2}{n+1}}, \quad d^{n-1} \approx \alpha^{2 n-2} \approx \Delta\|\nabla u\|_{\infty}^{n-3} .
$$

These last two estimates should be compared with Theorem 2.3 and with (5) in Theorem 1.2, respectively, in the case $n \geq 4$. 


\section{REFERENCES}

1. F. Bernstein, Über die isoperimetrische Eigenschaft des Kreises auf der Kugeloberfläche und in der Ebene, Math. Ann. 60 (1905), 117-136.

2. T. Bonnesen, Über die isoperimetrische Defizit ebener Figuren, Math. Ann. 91 (1924), 252268.

3. __ Les problèmes des isopérimètres et des isépiphanes, Gauthier-Villars, Paris, 1929.

4. T. Bonnesen and W.Fenchel, Theorie der konvexen Körper, Ergeb. Math., Bd. 3, Heft 1, Springer, Berlin, 1934, reprinted 1974; English transl., BCS Associates, Moscow, Idaho, 1987..

5. A. Dinghas, Bemerkung zu einer Verschärfung der isoperimetrischen Ungleichung durch $H$. Hadwiger, Math. Nachr. 1 (1948), 284-286.

6. B. Fuglede, Stability in the isoperimetric problem, Bull. London Math. Soc. 18 (1986), 599-605.

7. H. Hadwiger, Die isoperimetrische Ungleichung im Raum, Elemente Math. 3 (1948), 25-38.

8. A. Hurwitz, Sur quelques applications géométriques des séries de Fourier, Ann. Sci. École Norm. Sup. (3) 19 (1902), 357-408, Also in Mathematische Werke I, Birkhäuser, Basel, 1932, pp. 509-554..

9. C. Müller, Spherical harmonics, Lecture Notes in Math., vol. 17, Springer, Berlin, Heidelberg and New York, 1966.

10. R. Osserman, A strong form of the isoperimetric inequality in $\mathbf{R}^{n}$, Complex Variables, Theory and Application 9 (1987), 241-249.

Mathematical Institute, University of Copenhagen, Universitetsparken 5, 2100 Copenhagen $\emptyset$, Denmark 